\title{
A BCU Method for Direct Analysis of Power System Transient Stability
}

\author{
Hsiao-Dong Chiang \\ School of Electrical Engineering \\ Cornell University \\ Ithaca, NY 14853
}

\begin{abstract}
A boundary of stability region based controlling unstable equilibrium point method (BCU method) for direct analysis of power system transient stability is presented. Features distinghishing the $\mathrm{BCU}$ method from the existing direct methods are that it consistently finds the exact controlling u.e.p. relative to a fault-on trajectory and that it has a sound theoretical basis. Moreover, the BCU method appears to be fast. This method is based on the relationship between the boundary of stability region of a power system and that of a reduced system. The BCU method finds the controlling u.e.p. of the original system via a reduced system whose controlling u.e.p. is easier and cheaper to compute. Effective numerical schemes to speed up the presented method are also proposed. This method has been tested on several power systems with very promising results. Simulation results on a 50-generator, 145-bus system are presented along with a comparison between the results obtained using the $\mathrm{BCU}$ method and another existing method.
\end{abstract}

\section{Introduction}

Transient stability analysis plays an important role in the planning and operation of electric power systems. Until recently, transient stability analysis has been performed by utilities exclusively by means of the numerical integration of nonlinear differential equations describing the fault-on system and the post-fault system. An alternative approach to transient stability analysis employing Lyapunov function theory, called the direct methods, was proposed in $1966[1,2]$. The growing need for tools suitable for dynamic security assessment, as well as for a fast screening tool for planning stability studies, has generated renewed interests in such direct methods [3-5]. In this paper, direct methods refer to those methods that determine the stability of a post-fault system based on energy functions without explicitly integrating differential equations describing the post-fault system.

Recently, significant progress has been made in the direct methods for transient stability analysis. The details may be found, for example, in [6-11]. Among the direct methods, the classical method using the concept of closest u.e.p. (unstable equilibrium point) gives very conservative estimates of

91 SM 423-4 PWRS A paper recommended and approved by the IEEE Power System Engineering Committee of the IEEE Power Engineering Society for presentation at the IEEE/PES 1991 Summer Meeting, San Diego, California, July 28 - August 1, 1991. Manuscript submitted February 1, 1991; made available for printing June 18, 1991.

\author{
Felix F. Wu and Pravin P. Varaiya \\ Department of EECS \\ University of California \\ Berkeley, CA 94720
}

stability because this method is independent of the fault location. The potential energy boundary surface (PEBS) method proposed by Kakimoto et al. [16] gives fairly fast and accurate stability assessments if it works. This method may give inaccurate (both over-estimate and under-estimate) results [26]. A fault-dependent method using the concept of controlling u.e.p. (relevant u.e.p.) makes the direct methods more applicable in practical systems [17]. It is believed that the controlling u.e.p. method will continue to be a viable method, in terms of its accuracy and reliability, among the direct methods for transient stability analysis. The issue in the controlling u.e.p. method is how to find the precise controlling u.e.p. relative to a fault-on trajectory.

In $[14,30]$, we have developed a comprehensive theory of stability regions for general nonlinear dynamical systems. Applying these results to power system transient stability analysis, we have provided a theoretical foundation for the direct methods in [14] and for the PEBS method in [31]. In this paper, we first present a result to show that the controlling u.e.p. method analyzed in [14] also hold for any power system model having an energy function even without the assumption of the transversality condition. We then present a boundary of stability region controlling unstable equilibrium point method (BCU method) for direct analysis of power system transient stability. To make the method more effective, several computational schemes are developed and incorporated into the method. This method is based on the relationship between the boundary of stability region of a power system and that of a reduced system. Features distinghishing the $\mathrm{BCU}$ method from the existing direct methods are that it consistently finds the exact controlling u.e.p. relative to a fault-on trajectory and that it has a sound theoretical basis. This method has been tested on several power systems with very promising results.

\section{Preliminary}

A power system model (for transient stability analysis) is said to be transiently stable for a particular (pre-fault) steady-state operating condition and for a particular disturbance if, following that disturbance, the system reaches an acceptable steady-state operating condition (i.e. a desired stable equilibrium point of a post-fault system). Transient stability analysis essentially determines whether the faulton trajectory at clearing time lies inside the stability region (domain of attraction) of a desired stable equilibrium point (s.e.p.) of its post-fault system. Accordingly, knowledge of the stability region of a stable equilibrium point is very important in transient stability analysis. 
To unify the notations for subsequent analysis, we let

$$
\dot{x}=f(x)
$$

be the power system model under study, and $\mathrm{x}$ the corresponding state vector. Suppose that $x$ is a (asymptotically) stable equilibrium point (s.e.p.) of system (1) (i.e. all the eigenvalues of the Jacobian matrix of $f(x)$ at $x$, have strictly negative real parts.), then there is a region $A\left(x_{s}\right)$ containing $x_{s}$ such that every trajectory in this region converges to the s.e.p. $x$, as time increases (goes to positive infinity). The region $A\left(x_{s}\right)$ is termed the stability region of $x_{s}$ and the boundary of the stability region $A\left(x_{s}\right)$, denoted by $\partial A\left(x_{s}\right)$, is called the stability boundary (or separatrix) of $x_{s}$.

Energy functions play an important role in direct methods for transient stability analysis. A function $V(x)$ is said to be an energy function for system (1) if it satisfies the following three conditions: (i) $\dot{V}(x(t)) \leq 0$, (i.e. energy functions are non-increasing along any trajectory), (ii) the set $\{t \in$ $R: \dot{V}(x(t))=0$ \} has measure zero in $\mathrm{R}$, for any non-trivial trajectory $x(t)$ and (iii) if $V(x(t))$ is bounded in positive time, then $x(t)$ is bounded in positive time. The first two properties imply that energy functions are strictly decreasing along system trajectories. The third property is important in the characterization of stability boundary of system (1).

We next present some analytical results on characterizing a stability boundary of system (1). Theorem 1 below describes a global behavior of system trajectories on the stability boundary. A corollary of Theorem 1 offers a characterization of the stability boundary.

Theorem 1:[14] (The behavior of trajectories on the stability boundary)

If there exists an energy function for system (1), then every trajectory on a stability boundary, say $\partial A\left(x_{s}\right)$ converges to one of the equilibrium points on $\partial A(x$,$) as time increases..$

Theorem 1 indicates that every trajectory on a stability boundary of system (1) has only one mode of behavior: it converges to an equilbrium point as time increases. There does not exist any limit cycle (oscillating behavior) or bounded complicated behavior, such as a-periodic trajectory, chaotic trajectory, or un-bounded trajectory (going to infinity) on stability boundaries of system (1) provided an energy function exists. This theorem also offers a way to characterize the stability boundary. In fact, Theorem 1 implies that the stability boundary $\partial A\left(x_{0}\right)$ is composed of several discrete 'objects': Each object consists of an unstable equilibrium point (u.e.p.), say $x_{i}$, and other points whose trajectory converges to $x_{i}$ as time increases (see Figure 1). In mathematical language, each object in the stability boundary is part of the stable manifold of $x_{i}$. Recall that the stable manifold of an equilibrium point is the set such that the trajectory of every point in this set converges to the equilibrium point as time increases [25].

Corollary 2: [12], [14] (Characterization of the stability boundary)

If there exists an energy function for system (1), then the stability boundary $\partial A\left(x_{s}\right)$ is contained in the set which is the union of the stable manifolds of the unstable equilibrium points on the $\partial A\left(x_{s}\right)$; i.e.

$$
\partial A\left(x_{s}\right) \subseteq \cup_{x_{i} \in \partial A\left(x_{*}\right)} W^{s}\left(x_{i}\right)
$$

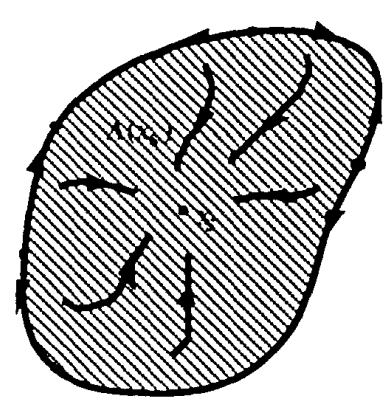

Figure 1: As time increases, every trajectory on the stability boundary $\partial A\left(x_{s}\right)$ converges to one of the equilibrium points on $\partial A\left(x_{s}\right)$ and every trajectory in the stability region $\mathrm{A}\left(x_{s}\right)$ converges to the s.e.p. $x_{s}$

\section{The Controlling U.E.P. Method}

The evolution of the concept of controlling u.e.p. can be traced back to the mid-1970s. In [18], Prabhakara and ElAbiad argued that the controlling u.e.p. is the u.e.p. which is "closest" to the fault-on trajectory. Athay, et al. in [17] suggested that the controlling u.e.p. is the u.e.p. "in the direction" of the fault-on trajectory. Another viewpoint of the controlling u.e.p. rested on physical arguments. RibbensPavella et al. [27] relate the controlling u.e.p. to the machine (or group of machines) which first go out of synchronism if the fault is sustained. Fouad et al. [20] associate the controlling u.e.p. with the "mode of instability" of machines.

We define the controlling u.e.p. with respect to a faulton trajectory $x_{f}(t)$ to be the u.e.p. whose stable manifold contains the exit point of $x_{f}(t)$ (see Fig. 2). This definition is based on the fact that the exit point (i.e. the point from which a given fault-on trajectory exits the stability boundary of the post-fault system) of a fault-on trajectory must lie on the stable manifold of some u.e.p. on the stability boundary. Note that Corollary 2 guarantees the existence and uniqueness of the controlling u.e.p. relative to a given fault-on trajectory.

The main purpose in the transient stability analysis is not to estimate the whole stability boundary of a stable equilibrium point of the post-fault system. Instead, only the relevant part of stability boundary to which the fault-on trajectory is heading is of main concern. When the closest u.e.p. method was applied to power system transient stability analysis, this method was found to yield conservative results. In fact, in the context of transient stability analysis, the closest u.e.p. method provides an approximation to the entire stability boundary of the post-fault system and is independent of the fault-on trajectory. Thus, the closest u.e.p. method gives conservative results for transient stability analysis. A desirable method for transient stability analysis would be one which can provide a more accurate approximation of the relevant part of the stability boundary to which the fault-on trajectory is heading, even though it might provide a poor estimate of the other part of the stability boundary of the 


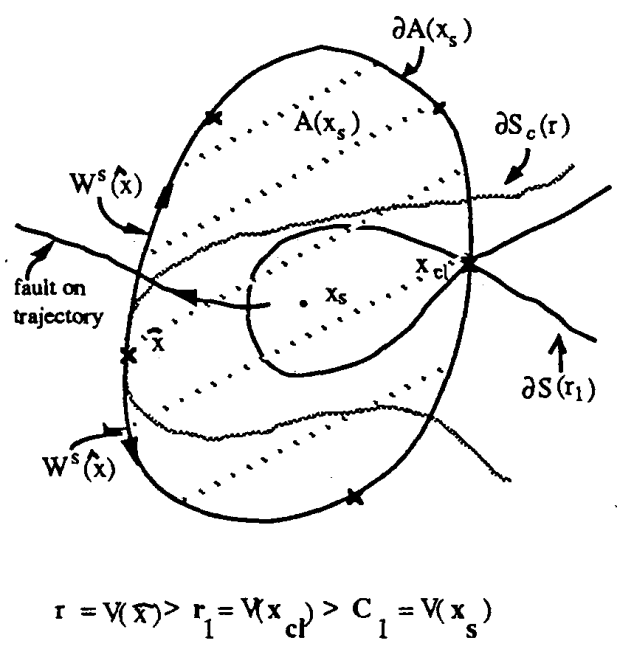

Figure 2: The fault-on trajectory $x_{f}(t)$ moves toward the stability boundary $\partial A\left(x_{s}\right)$ of the s.e.p. $x_{s}$ of a post-fault system. The exit point of this fault-on trajectory is the point $x_{e}$ which lies on the stable manifold of $\hat{x}$. The equilibrium point $\hat{x}$ is the controlling u.e.p. of the fault-on trajectory $x_{f}(t)$ and $x_{c l}$ is the closest u.e.p.

post-fault system. The controlling u.e.p. method belongs to this class of methods.

The controlling u.e.p. method uses the constant energy surface passing through the controlling u.e.p. to approximate the relevant part of the stability boundary to which the fault-on trajectory is heading. Theorem 3 below gives a theoretical justification of the controlling u.e.p. method. This theorem is an extension of Theorem 6-4 in [31] which relaxes the assumption of transversality intersection between the stable and unstable manifolds. The transversality intersection is a generic property but is difficult to check.

Theorem $3:$ (Fundamental theorem for the controlling u.e.p. method)

Suppose that a nonlinear system described by $\dot{x}=f(x)$, x $\in R^{n}$ has an energy function $V(\cdot): R^{n} \rightarrow R$. Let $\dot{x}$ be an equilibrium point on the stability boundary $\partial A\left(x_{s}\right)$ of this system. Let

- $S_{c}(r):=$ the connected component of the set $\{x: V(x)$ $<\mathrm{r}\}$ containing $x_{s}$, and

- $\partial S_{c}(r):=$ the connected component of the set $\{\mathrm{x}$ : $\mathrm{V}(\mathbf{x})=\mathbf{r}\}$ containing $x_{\mathbf{s}}$.

Then, the connected constant energy surface $\partial S_{c}(V(\hat{x}))$ intersects with the stable manifold $W^{s}(\hat{x})$ only at point $\hat{x}$; moreover, the set $S_{c}(V(\hat{x}))$ has an empty intersection with the stable manifold $W^{s}(\hat{x})$.

Remarks:

[1] Theorem 3 asserts that for any fault-on trajectory $x_{f}(t)$ starting from a point $p \in A\left(x_{3}\right)$ with $V(p)<V(\hat{x})$, if the exit point of the fault-on trajectory lies in the stable manifold of $\hat{x}$, then the fault-on trajectory must pass through the connected constant energy surface $\partial S_{c}(V(\hat{x}))$ before it passes through the stable manifold of $\hat{x}\left(W^{s}(\hat{x})\right.$ ) (thereby exiting the stability boundary $\left.\partial A\left(x_{s}\right)\right)$. Therefore, the connected constant energy surface $\partial S_{c}(V(\hat{x}))$ can be used to approximate the relevant part of the stability boundary $\partial A\left(x_{s}\right)$ for the fault-on trajectory $x_{f}(t)$.

[2] Theorem 3 indicates the inherent conservative property of the controlling u.e.p. method for estimating the critical energy value for the fault-on trajectory (but less conservative than the closest u.e.p. method). Figure 2 shows that the fault-on trajectory passes through first the constant energy surface of the closest u.e.p. $\left(x_{c l}\right)$, then the constant energy surface of the controlling u.e.p. $(\hat{x})$, and finally the stability boundary.

The controlling u.e.p. method for power system transient stability analysis proceeds as follows : Let $\mathrm{V}(\mathrm{x})$ be an energy function of a post-fault system and $x_{f}(t)$ be a fault-on trajectory.

A conceptual controlling u.e.p. method:

Step 1: Determine the controlling u.e.p. $x_{c o}$ for the fault-on trajectory $x_{f}(t)$.

Step 2: The critical energy $v_{c}$ is the value of energy function $V(\cdot)$ at the controlling u.e.p., i.e.

$$
v_{c}=V\left(x_{c o}\right)
$$

Step 3: Calculate the value of the energy function $V(\cdot)$ at the time of fault clearance (say, $t_{c l}$ )

$$
v_{f}=V\left(x_{f}\left(t_{c l}\right)\right) \text {. }
$$

Step 4: If $v_{f}<v_{c}$, then the post-fault system is stable. Otherwise, it is unstable.

The key element in the controlling u.e.p. method is how to find the controlling u.e.p for a fault-on trajectory. Without finding the (precise) controlling u.e.p. in step 1, the above method can give either an over-estimate or a under-estimate stability assessment. Generically, there is no other u.e.p. whose energy function value is the same as that of the controlling u.e.p.

Due to the importance of finding the (correct) controlling u.e.p., our next objective is to derive a theory-based algorithm for finding the controlling u.e.p. relative to a fault-on system. An algorithm to find the controlling u.e.p. for the classical power system model with transfer conductance will be presented in the sequel.

\section{System Model}

We review the classical model for transient stability analysis. Consider a power system consisting of $n$ generators. Let the loads be modeled as constant impedances. The dynamics of the $\mathrm{i}$-th generator can be represented by the following equations

$$
\begin{aligned}
\dot{\delta}_{i} & =\omega_{i} \quad i=1,2, \ldots, n \\
M_{i} \dot{\omega}_{i} & =P_{i}-D_{i} \omega_{i}-P_{e i}
\end{aligned}
$$

where node $n+1$ serves as the reference node, i.e. $E_{n+1}=1$ and $\delta_{n+1}=0 . E_{i}$ is the constant voltage behind direct axis transient reactance. $P_{e i}=\sum_{j \neq i}^{n+1} E_{i} E_{j} B_{i j} \sin \left(\delta_{i}-\delta_{j}\right)+$ 
$\sum_{j \neq i}^{n+1} E_{i} E_{j} G_{i j} \cos \left(\delta_{i}-\delta_{j}\right) . M_{i}$ is the generator's moment of inertia. The damping constant $D_{i}$ is assumed to be positive. $G_{i j}$ term represents the transfer conductance of the $i-j$ element in the reduced admittance matrix of the system. $P_{i}$ $=P_{m i}-E_{i}^{2} G_{i i}$, where $P_{m i}$ is the mechanical power. We assume uniform damping $\frac{D_{i}}{M I_{i}}=\lambda, \mathrm{i}=1,2, \ldots, \mathrm{n}$.

The equations describing the pre-fault, fault-on and postfault systems all have the same form as (4) except $G_{i j}^{\prime} s$ and $B_{i j}^{\prime} s$ are different due to change in network topology.

Using one machine as reference, equation (5) can be transformed into the following:

$$
\begin{aligned}
& \dot{\delta_{i n}}=\omega_{i n} \quad i=1,2, \ldots, n-1 \\
& \dot{\omega}_{i n}=\frac{1}{M_{i}}\left(P_{i}-P_{e i}\right)-\frac{1}{M_{n}}\left(P_{n}-P_{e n}\right)-\lambda \omega_{i n}
\end{aligned}
$$

$\delta_{i n}=\delta_{i}-\delta_{n}, \omega_{i n}=\omega_{i}-\omega_{n}$.

One (numerical) energy function for system (5) is of the following form [17] with $M_{T}=\sum_{i=1}^{n} M_{i}$ :

$$
\begin{array}{r}
V(\delta, \omega)=\sum_{i=1}^{n-1} \sum_{j=i+1}^{n}\left(\frac{1}{2 M_{T}} M_{i} M_{j}\left(\omega_{i n}-\omega_{j n}\right)^{2}\right. \\
-\frac{1}{M_{T}}\left(P_{i} M_{j}-P_{j} M_{i}\right)\left(\delta_{i n}-\delta_{j n}-\delta_{i n}^{s}+\delta_{j n}^{s}\right) \\
-V_{i} V_{j} B_{i j}\left(\cos \left(\delta_{i n}-\delta_{j n}\right)-\cos \left(\delta_{i n}^{s}-\delta_{j n}^{s}\right)\right) \\
-V_{i} V_{j} G_{i j} \frac{\delta_{i n}+\delta_{j n}-\left(\delta_{i n}^{s}+\delta_{j n}^{s}\right)}{\delta_{i n}-\delta_{j n}-\left(\delta_{i n}^{s}-\delta_{j n}^{s}\right)} \\
\\
\left(\sin \left(\delta_{i n}-\delta_{j n}\right)-\sin \left(\delta_{i n}^{s}-\delta_{j n}^{s}\right)\right) \\
=V_{p}(\delta)+\frac{1}{2 M_{T}} \sum_{i=1}^{n-1} \sum_{j=1}^{n} M_{i} M_{j}\left(\omega_{i n}-\omega_{j n}\right)^{2}
\end{array}
$$

It snould be pointed out that, theoretically speaking, the controlling u.e.p. method should give stability estimates on the conservative side if an exact energy function exists. Recall that an exact energy function is a well-defined function and its function value is strictly decreasing along any trajectory of the system [14]. It has, however, been shown that there does not exist a general form of exact energy function for power systems with transfer conductances [29]. The existing numerical energy functions are derived on the assumption that the fault-on trajectory is close to a straight line. The assumption is widely received in the area of direct methods. It should, however, be cautioned that the validity of this assumption may affect the property of the controlling u.e.p. method: it always gives stability estimates on the conservative side.

\section{The BCU Method}

In this section we describe the BCU method to find the controlling u.e.p. for the classical power system model. Even though the classical model is written in the last section in terms of the reference machine coordinate, the BCU method works as well for the center-of-angle coordinate. The method is based on the relationship between the stability boundary of the (post-fault) classical power system model (5) and the stability boundary of the following (post-fault) reduced system [31]:

$$
\begin{array}{rlrl}
\dot{\delta}_{i n} & =\left(P_{i}-P_{e i}\right)-\frac{M_{i}}{M_{n}}\left(P_{n}-P_{e n}\right) \\
& =f_{i}(\delta) & i=1,2, \ldots, n-1
\end{array}
$$

The state variables of the reduced system (7) are machine angles only with dimension of $n-1$ while the dimension of the original system (5) is of $2(\mathrm{n}-1)$. It can be seen that $(\hat{\delta})$ is an equilibrium point of the reduced system (7) if and only if $(\hat{\delta}, 0)$ is an equilibrium point of the original system. Furthermore, under the condition of small transfer conductances, it can be shown that

(RI) $\left(\hat{\delta}_{s}\right)$ is a stable equilibrium point of the reduced system (7) if and only if $\left(\hat{\delta}_{s}, 0\right)$ is a stable equilibrium point of the original system (5).

(R2) $\left(\bar{\delta}_{x}\right)$ is a type-k equilibrium point of the reduced system $(7)$ if and only if $\left(\hat{\delta}_{s}, 0\right)$ is a type-k equilibrium point of the original system (5).

(R3) If the one-parameter transversality condition is satisfied, then $(\bar{\delta})$ is on the stability boundary $\partial A\left(\hat{\delta}_{s}\right)$ of the reduced system $(7)$ if and only $(\bar{\delta}, 0)$ is on the stability boundary $\partial A\left(\hat{\delta}_{s}, 0\right)$ of the original system $(5)$.

A proof of these results under the condition of zero transferconductance is contained in [31]. Result (R1) reveals that it makes sense to compare the stability region $A\left(\hat{\delta}_{0}\right)$ of the reduced system $(7)$ with the stability region $A\left(\hat{\delta}_{s}, 0\right)$ of the original system (5). Result (R3) establishes a relationship between the stability boundary $\partial A\left(\hat{\delta}_{s}\right)$ and the stability boundary $\partial A\left(\hat{\delta}_{s}, 0\right)$ and suggests the plausibility of finding the controlling u.e.p. of the original system (5) via finding the controlling u.e.p. of the reduced system (7).

The $B C U$ method : find the controlling u.e.p. relative to a fault-on trajectory (version 1)

Step 1: From the fault-on trajectory $(\delta(t), \omega(t))$, detect the exit point $\delta^{*}$ which is the point the projected trajectory $\delta(t)$ exits the stability boundary of the reduced system (7).

Step 2: Use the point $\delta^{*}$ as the initial condition and integrate the post-fault reduced system (7) to find the first local minimum of $\sum_{i=1}^{n}\left\|f_{i}(\delta)\right\|$, say at $\delta_{o}^{*}$.

Step 3: Use the point $\delta_{0}^{*}$ as the initial guess to solve $\sum_{i=1}^{n}\left\|f_{i}(\delta)\right\|=0$, say at $\delta_{c o}^{*}$.

Step 4: Assign the controlling u.e.p. with respect to the faulton trajectory to be $\left(\delta_{c o}^{*}, 0\right)$.

Once the controlling u.e.p. is found, the BCU method uses the same procedure as that of the conceptual controlling u.e.p. method presented in section 4 (step 2 - step 4) to perform stability assessment. The essence of the BCU method is that it finds the controlling u.e.p. via the controlling u.e.p. of the reduced system (7) which is defined in the angle space only and whose controlling u.e.p. is easier to compute. Steps 1-3 find the controlling u.e.p $\boldsymbol{p}_{\text {n }}$ of the reduced system and step 4 relates the controlling u.e.p. of the reduced system to the controlling u.e.p. of the original system (6).

\section{Remarks:}

[1] An effective computation scheme to implement Step 1: From the fault-on trajectory $(\delta(t), \omega(t))$, detect the exit point $\delta^{*}$ at which the projected trajectory $\delta(t)$ reaches the first local maximum of $V_{p}(\cdot)$.

[2] The reduced system (7) could be stiff. In such a case, a stiff differential equation solver is recommended to implement Step 2. 
[3] While the proposed method uses the potential energy at the controlling u.e.p. of the reduced system, $\delta_{c o}$, as the critical energy value, the PEBS method uses the potential energy at $\delta^{*}$ as the critical energy value. An explanation of why the PEBS method may give both over-estimate and under-estimate stability results is given in [31].

[4] Several existing direct methods can be viewed as finding the controlling u.e.p. in the angle space. For example, Fouad et al. find the controlling u.e.p. via the "mode of disturbance" of machines. Pavella et al. locate the controlling u.e.p. via the "accelerating machines." The proposed algorithm finds the controlling u.e.p. of the reduced system (in the angle space) via two steps. The first step locates the exit point of the reduced system. Recall from Theorem 1 that the exit point must lie on the stable manifold of the controlling u.e.p. Hence, the second step integrates the reduced system starting from the exit point and the resulting trajectory will converge to the controlling u.e.p. as time increases (In practical computation, the point calculated in Step 1 cannot be exactly the exit point but lies in a neighborhood of the exit point. Consequently, the resulting trajectory will just pass by the controlling u.e.p.)

[5] We strongly believe that the essence of the $\mathrm{BCU}$ method can be extended to develop solution algorithms for transient stability analysis of detailed power system models.

\section{Computational Considerations}

There are basically three major computational tasks in the proposed solution algorithm: (1) compute the point with the first local maximum of potential energy along the projected fault-on trajectory, (2) compute the trajectory of the postfault reduced system and (3) compute the controlling u.e.p. via solving the nonlinear algebraic equations related to the reduced system. We discuss in this section some schemes to speed up the proposed solution algorithm.

From a computational point of view, it is very difficult to compute the exact point where the first local maximum of potential energy along the projected fault-on trajectory occurs. This is because we simulate the trajectory on a digital computer which yields only a sequence of points but not the entire trajectory. Hence, step 1 of the proposed solution algorithm usually gives a point which is close to the exit point. In order to speed up the computation without sacrificing accuracy, the following solution algorithm is recommended when a large integration step size is used in integrating the fault-on system.

\section{The BCU method (version 2)}

Step 1: From the fault-on trajectory $(\delta(t), \omega(t))$, detect the point $\delta^{*}$ at which the projected trajectory $\delta(t)$ reaches the first local maximum of $V_{p}(\cdot)$. Also, compute the point $\delta^{+}$that is one step after $\delta^{*}$.

Step 2: Use the point $\delta^{*}$ as initial condition and integrate the post-fault reduced system to find the first local minimum of $\sum_{i=1}^{n}\left\|f_{i}(\delta)\right\|$, say at $\delta_{0}^{*}$.

Step 3: Use $\delta^{+}$as an initial condition and repeat Step 2 to find the corresponding points, say $\delta_{0}^{+}$.
Step 4: Compare the values of $\|f(\delta)\|$ at $\delta_{o}^{*}$ and $\delta_{o}^{+}$. The one with the smallest value is used as the initial guess to solve $(7), f_{i}(\delta)=0$, say the solution is $\delta_{c o}$.

Step 5: The controlling u.e.p. with respect to the fault-on trajectory is $\left(\delta_{c o}, 0\right)$.

Note that the point $\delta^{*}$ of step 1 at which the projected trajectory $\delta(t)$ reaches the first local maximum of $V_{p}(\cdot)$ may not accurately be the point at which the first local maximum of $V_{p}(\cdot)$ occurs. That is the reason we compute the other two points at step 2 .

A large integration step size used in step 1 may cause the first local minimum in Step 2 not to be found in the simulation. In this case, one should go back to Step 1 and take a smaller step size to detect the new points $\delta^{*}, \delta^{+}$and repeat Step 2 to Step 5.

\section{Test Results}

The proposed method has been tested on several power systems. In this section we present test results on a 50generator, 145-bus system. We have applied the proposed method to several three-phase faults with fault locations at both generator and load buses. The numerical energy function (6) is used in the simulations. Both severe and mild faults have been considered. In all the cases, the pre-fault system is different from the post-fault system. For example, a fault occuring at bus 6 causes the circuit breakers on the line between buses 6 and 12 to open to clear the fault.

Table 1 displays the estimated critical clearing time of several faulted systems using four different methods: stepby-step numerical integration technique, the BCU method (version 2), the BCU method (version 2) with corrected kinetic energy [20] and the MOD method [8]. The results from the numerical integration technique are used as a benchmark. A few observations and comments on the simulation results follow.

The BCU method consistently found the exact controlling u.e.p. The PEBS method is fast but is inconsistent in accuracy. There are a few cases for which the MOD method did not converge to any u.e.p. And in some cases, the MOD method converges to a u.e.p. which is not a controlling u.e.p. In all but one case, the BCU method gives slightly conservative results in CCT (under-estimate). This is consistent with the results derived in Theorem 3 . The only case that the $\mathrm{BCU}$ method gives an over-estimate is when a fault occurs at bus 58. In this case, the CCT from benchmark is $0.51 \mathrm{sec}$. while the estimated CCT by the BCU method is $0.59 \mathrm{sec}$. This case was then further studied and we found that the BCU method still finds the correct controlling u.e.p. However, a whole group of machines in this case tend to become unstable such that the assumption in deriving the energy function that the fault-on trajectory is a straight line does not hold. Thus, it is the accuracy of the energy function (i.e. the conditions required for energy functions are not satisfied) for this case that causes the over-estimate. It has been our experience that those cases involving a whole group of machines tend to be unstable and usually damage the accuracy of energy functions.

For those cases that the MOD method converges, the estimated CCT's are fairly good even though the u.e.p. found 


\begin{tabular}{|c|c|c|c|c|c|c|}
\hline \multirow[b]{2}{*}{$\begin{array}{l}\text { Fault location } \\
\text { at bus }\end{array}$} & \multirow[b]{2}{*}{$\begin{array}{l}\text { Line clearing } \\
\text { between buses }\end{array}$} & \multicolumn{5}{|c|}{ Critical Clearing Time estimated by different methods } \\
\hline & & $\begin{array}{l}\text { Step-by-Step } \\
\text { simulation } \\
\text { Unit: sec }\end{array}$ & $\begin{array}{c}\text { The BCU Method } \\
\text { Unit: sec }\end{array}$ & $\begin{array}{l}\text { The BCD Method } \\
\text { + corrected energy } \\
\text { Unit: sec }\end{array}$ & $\begin{array}{c}\text { The PEBS Method } \\
\text { Unit:sec }\end{array}$ & $\begin{array}{c}\text { The MOD Method } \\
\text { Unit:sec }\end{array}$ \\
\hline 6 & $6-12$ & 0.200 & 0.170 & 0.185 & 0.180 & $* * *$ \\
\hline 7 & $6-7$ & 0.119 & 0.115 & 0.122 & $0.1 \overline{13}$ & 0.113 \\
\hline 12 & $12-14$ & 0.190 & 0.170 & 0.190 & 0.180 & $\$ \$$ \\
\hline 58 & $58-87$ & 0.51 & 0.59 & - & 0.395 & F**; \\
\hline 63 & $61-63$ & 0.91 & 0.855 & 0.91 & 0.565 & $7 \neq$ \\
\hline 90 & $90-92$ & 0.270 & 0.265 & 0.270 & 0.275 & $7 * 7$ \\
\hline 96 & $96-73$ & 0.245 & 0.242 & 0.245 & 0.240 & 0.240 \\
\hline 97 & $97-66$ & 0.260 & 0.260 & 0.260 & 0.245 & 0.250 \\
\hline 98 & $98-72$ & 0.205 & 0.205 & 0.205 & $0.1 \overline{95}$ & 0.190 \\
\hline 100 & $100-72$ & 0.315 & 0.315 & 0.320 & $0 . \overline{305}$ & 0.315 \\
\hline 102 & $63 \cdot 102$ & 0.200 & 0.195 & 0.195 & 0.185 & 0.195 \\
\hline 116 & $116-63$ & 0.28 & 0.28 & 0.28 & 0.265 & 0.285 \\
\hline
\end{tabular}

Table 1: The Critical Clearing Time. The sign ${ }^{* * *}$ means that the method does not converge to any U.E.P.

by the method may not be the controlling u.e.p. The simulation results also reveal that the MOD method gives both slight over-estimates and under-estimates. This is probably due to the fact that the MOD method is heuristic in nature and in this method a threshold value is required to determine the sorting list in order to find the controlling u.e.p. If the threshold value is not properly chosen, two cases can happen. Case 1: The threshold value is chosen higher than the (exact) threshold value so that the machines corresponding to the controlling u.e.p. are not all included in the sorting list. In this case, the MOD method finds a u.e.p. other than the controlling u.e.p. Case 2: The threshold value is chosen lower than the (exact) threshold value. In this case, the u.e.p. with the lowest normalized potential margin at the instant the disturbance is removed is very unlikely to be the controlling u.e.p. It is observed from this table that the proposed BCU method offers fairly accurate direct analysis of transient stability for these faults.

The BCU method in conjunction with the corrected kinetic energy method gives on the average very good results except sometimes they together give over-estimate results such as the faults occurring at bus \#7 and bus \#100. In all the other cases, they reduce the conservativeness of the BCU method. This indicates that the corrected kinetic energy method can be quite effective and deserves further (theoretical) investigation.

It can be argued that for those cases that the MOD method does not converge, a fine-tuned procedure on the threshold value in a case-by-case basis can make the method converge. But, this fine-tuned procedure has to be executed on a case-by-case procedure and there is no systematic guideline as to how to exercises the fine-tuned procedure. Furthermore, it may again converge to a U.E.P. other than the controlling U.E.P.

\section{Conclusion}

We have presented a boundary of stability region based controlling u.e.p. (BCU) method for direct analysis of transient stability. The BCU method is based on the relationship between the stability boundary of the classical power system model and the stability boundary of a reduced system which is defined in the angle space only. The essence of the BCU method is that it finds the controlling u.e.p. via the controlling u.e.p. of the reduced system which is defined in the angle space and whose controlling u.e.p. is easier to compute. Effective numerical schemes to speed up the presented controlling u.e.p. method are proposed. This method has been tested on several power systems with very promising results.

We strongly believe that this essence can be extended to develop solution algorithms for transient stability analysis of detailed power system models. Research work is still being done to extend the BCU method to power systems where a structure-preserving network, a more realistic load model and a detailed generator model are used.

\section{Acknowledgement}

The BCU method presented in this paper has been tested on several power systems by researchers at Iowa State University, University of Illinois and Ontario Hydro, among others. The authors wish to express appreciation to them for their insightful comments and for sharing interesting observations with them. The authors gratefully acknowledge support in part from NSF under grant numbers ECS-8810544, ECS8957878 and ECS-8913074.

\section{References}

[1] G.E. Gless, "Direct method of Lyapunov applied to transient power system stability," IEEE Trans. on Power Apparatus and Systems, Vol.PAS-85, Feb., 1966, pp.159-168.

[2] A.H. El-Abiad and K. Nagappan, "Transient stability regions for multi-machine power systems," IEEE Trans. on Power Apparatus and Systems, Vol. PAS-85, Feb., 1966, pp.169-179.

[3] F.F. Wu and Y-K. Tsai, "Probabilistic dynamic security assessment of power systems, Part I: Basic model," IEEE Trans. on Circuits and Systems Vol. CAS-30, March ,1983, pp.148-159.

[4] R.J. Kaye and F.F. Wu, "Dymamic security regions for power systems," IEEE Trans. on Circuits and Systems Vol. CAS29, September, 1982, pp.612-623.

[5] IEEE Committee Report, "Dynamic Security Assessment Practices in North America" IEEE Trans. on Power Systems Vol. 3, 1988, pp.1310-1321.

[6] M. Ribbens-Pavella and F.J. Evans, "Direct methods for studying dynamics of large-scale electric power systems - A Survey," Automatica Vol-32, January, 1985, pp.1-21.

[7] P.P. Varaiya, F.F. Wu and R-L Chen, "Direct methods for transient stability analysis of power systems: Recent results," Praceedings of the IEEE, December 1985, pp. 1703-1715.

[8] A.A. Fouad and V. Vittal, "The transient energy function method", International Journal of Electrical Power and Energy Systems, Vol. 10, No. 4, Oct. 1988, pp. 233-246. 
[9] M.A. Pai, Energy Function Analysis for Power System Stability. Kluwer Academic Publishers, 1989.

[10] IEEE Committee Report, "Application of direct methods to transient stability analysis of power systems", IEEE Transaction on Power Apparatus and System, Vol. PAS-103, July, 1984.

[11] F. Mercede, R. Fischl, F.F. Wu and H.D. Chiang, “A Comparison of Dynamical Security Indices Based on Direct Methods", International Journal of Electrical Power and Energy Systems, Vol. 10, No. 4, Oct. 1988, pp. 210-232

[12] N. Tsolas, A. Arapostathis and P.P. Varaiya, "A structure preserving energy function for power system transient stability analysis," IEEE Trans. on Circuits and Systems Vol CAS-32, Oct. 1985, pp. 1041-1049.

[13] G.A. Maria, C. Tang and J. Kim, "Hybrid transient stability analysis", IEEE Transaction on Power Systems, Vol. 5, No. 2, May, 1990, pp. 384-393.

[14] H.D. Chiang, F.F. Wu and P. P. Varaiya, "Foundation of Direct Methods for Power System Transient Stability Analysis", IEEE Trans. on Circuits and Systems Vol. CAS-34, Feb. 1987, pp. 712- 728.

[15] M.A. El-Kady, A.A. Fouad, et al., "Direct analysis of transient stability for large power systems" EPRI Report EL4980, RP2206-1, Dec. 1986.

[16] N. Kakimoto, Y. Ohsawa and M. hayashi, "Transient Stability Analysis Of Electric Power System via Lure-Type Lya punov Function, Part I and IN", Trans. IEE of Japan, Vol-98, pp.516, 1978 .

[17] T. Athay, R. Podmore and S. Virmani, "A practical method for direct analysis of transient stability", IEEE Transaction on Power Apparatus and System, Vol. PAS-98, 1979, pp.573584 .

[18] F.S. Prabhakara and A.H. El-Abiad, "A simplified determination of stability regions for Lyapunor method", IEEE Transaction on Power Apparatus and System, Vol. PAS-94, 1975, pp. 672-689.

[19] A.A. Fouad and V. Vittal, Power System Transient Stability Analysis Using the Transient Energy Function Method, to appear.

[20] A.A Fouad and S.E. Stanton, "Transient stability of a multimachine power systems. Part I :Investigation of system tra jectories" IEEE Transaction on Power Apparatus and System, Vol. PAS-100, 1981, pp. 3408-3414.

[21] "Proposed Terms and Definitions for Power System Stability", Task Force on Terms and Defunition, System Dynamic Performance Subcommittee, Power System Engineering Committee, IEEE Transaction on Power Apparatus and System, Vol.PAS-101, July, 1982, pp. 1894-1898.

[22] P.W. Sauer, A.K. Behera, M.A. Pai, J.R. Winkelman and J.H. Chow, "Trajectory approximations for direct energy methods that use sustained faults with detailed power system models", IEEE Transaction on Power System, Vol. 4 , No. 2, May 1989, pp. 499-506.

[23] A.S. Debs and A.R. Benson, "Security assessment of power systems", System Engineering for Power: Status and prospects, Proc. Eng. Foundation Conf., Ed. by L.H. Fink and K. Carlsen, Henniker, N.H., Aug. 1975, pp.144-176.

[24] W.W. Lemmon, K.R.C. Mamandur and W.R. Barcelo, "Transient stability prediction and control in real-time by QUEP", IEEE Transaction on Power System, Vol. 4, No. 2, May 1989, pp. 627-642.

[25] J. Guckenheimer and P. Holmes, Nonlinear Oscillations, Dynamical Systems, and Bifurcation Of Vector Fields, Springer-Verlag, 1983
[26] B. Toumi, R. Phifaoui, Th. Van Cutsem and M. RibbensPavella, "Fast Transient Stability Assessment Revisited", IEEE Trans. on Power Systems Vol. 1, 1986, pp.211-220.

[27] M. Ribbens-Pavella, P.G. Murthy, and J.L. Horward, "The acceleration approach to practical transient stability domain estimation in power systems," Proc. of the 20th IEEE Conference on Decision and Control, San Diego, CA, Dec. 16-18, 1981, pp. $471-477$.

[28] .H. Woodson and J.R. Melcher, Electromechanical Dynamics Part I: Discrete Systems, John Wiley, 1968.

[20] H.D. Chiang, "Study of the existence of energy functions for power system with losses", IEEE Trans. on Circuits and Systems Vol. CAS-36, Nov, 1989, pp. 1423-1429.

[30] H.D. Chiang, M. Hirsch and F.F. Wu, "Stability Regions of Nonlinear Autonomous Dynamical Systems", IEEE Trans. on Aatomatic Control, Vol. AC-33, pp. 14-27, Jan. 1988.

[31] H.D. Chiang, F.F. Wu and P. Varaiya, "Foundation of PEBS Method for Power System Transient Stability Analysis", IEEE Trans. on Circuits and Systems Vol. CAS-35, June 1988, pp. 712- 728 .

\section{Biography}

Hsiao-Dong Chiang received the B.S and M.S. degrees from the National Taiwan University, Taipei, Taiwan, and the Ph.D. degree in electrical engineering and computer sciences from the University of California at Berkeley in 1986, and then worked at the Pacific Gas and Electricity Company on a special project. He joined the Cornell faculty in 1987. He was a recipient of the Engineering Research Initiation Award (1988) and of the Presidential Young Investigator Award (1989) from the National Science Foundation. His research interests include power systems, nonlinear systems, optimization theory and neural networks. He is currently an Associate Editor of the IEEE Transactions on Circuits and Systems

Felix F. Wu (S'69-M'73-SM'86-F'89) received the $\mathrm{Ph} . \mathrm{D}$. degree in electrical engineering and computer sciences from the University of Califomia, Berkeley. He joined the Faculty at Berkeley in 1974 where he is presently Professor of Electrical Engineering and Computer Sciences. He has been a consultant to Pacific Gas and Electric Co. Electric Power Research Institute and other private companies. Dr. Wu has served on the IEEE Prize Paper Committee(1984-87), Editorial Board of the Proceedings of the IEEE (1983-87), and as an Associate Editor of the IEEE Transactions on Circuits and Systems (1981-83).

Pravin P. Varaiya (M'68-SM'78-F'80) received the B.E. degree from the University of Bombay, India, and the Ph.D. degree from the University of California, Berkeley. Since 1966, he has been with the University of California, Berkeley, where he is currently a Professor of Electrical Engineering \& Computer Sciences and Economics. His research interests are in the area of stochastic systems and urban economics. He is the coauthor (with P. R. Kumar) of Stochastic Systems: Estimation, Identification, and Adaptive Control, Englewood Cliffs, NJ: Prentice-Hall, 1986. 
Discussion

M. A. Pai (Dept. of Electrical Engineering, University of Illinois, Urbana, IL): The paper is an important contribution to the literature. It theoretically characterizes the multi-dimensional separatrix for the n-machine power system and for computational purposes uses the post-fault gradient system (eq. 7) to compute the controlling u.e.p after finding the PEBS crossing. Hence two "one-shot" integrations are involved, one for the faulted system up to the PEBS crossing and then for the gradient system (reduced system) to find the controlling u.e.p. The correction to K.E (version 2) removes the energy due to intermachine oscillations that does not contribute to system separation. It is therefore a nice blend of the earlier work in the area [16, $17,20]$.

The discussor has the following comments and questions.

1. In the energy function (6), the path dependent integral due to $G_{i j}$ terms can be evaluated by trapezoidal integration [17]. The authors use a straight line path which may lend to errors in computing PEBS crossing.

2. Did they assume any particular value for $\lambda$ (uniform damping)?

3. The use of rotor angles in the formulation forces one to choose a machine as reference. Use of center of angle (COA) would have streamlined the equations as well as interpretation of stability in terms of swing curves. To what extent does COA formulation affect the theoretical framework?

4. We have used the author's method (with $\lambda=0$ ) using COA formulation and using path dependent integrals. The results were very good. The straight line path is used only for computing $V_{c r}$ as in Ref. [17]. The kinetic energy can be corrected by noting the critical group(s) at PEBS crossing.

5. Can the authors give a physical explanation to the gradient equation since in equation (7) we have rotor speed one side of the equation and power on the other side. The explanation in their earlier work was very mathematical.

6. The discussor would like to know which faults in Table 1 resulted in a single $\mathrm{m} / \mathrm{c}$ going unstable and which ones resulted in a group of machines going unstable.

7. The PEBS method is known to give reliable results with structure preserving model and detailed dynamics $[22, \mathrm{~A}]$.

\section{Reference}

[A] K. R. Padiyar and K. K. Ghosh, "Direct stability evaluation of power systems with detailed generator models using structure preserving energy functions," International Journal of Electric Power and Energy Systems, vol. 11, no. 1, Jan. 1989, pp. 47-56.

K. R. Padiyar (Electrical Engineering Department, Indian Institute of Science, Bangalore, India): The authors are to be commended for a clear exposition of the BCU method derived from theoretical foundations for the computation of stability boundary near a fault-on trajectory. From the numerical results also, the method appears to be superior to MOD method.

While I look forward to the extension of the BCU method to structure preserving energy functions (SPEF), our experience shows that the PEBS method is quite accurate in such cases $(\mathrm{A}, \mathrm{B})$. The inaccuracies of the PEBS method as given in the paper, are in my view due to path dependent terms (or approximations) in the energy functions due to transfer conductances. Even with constant impedance loads, it is our experience that SPEF gives better results than the one defined on the system with the network reduced to the generator internal modes. The corrected kinetic energy method can also be applied to PEBS approach and improves accuracy of the predicted critical clearing times.

With structure preserving models, the computations of controlling u.e.p would be cumbersome and time consuming. The problems would increase with the detailed generator models. Would the authors like to comment on this?

It is well known that the inclusion of nonlinear load models result in significant differences as compared to linear (constant impedance) loads, while classical generator models are adequate approximations in estimating first swing stability. Hence I find that it is quite surprising that SPEF has not received adequate attention in the applications of direct methods.

As a minor point, I would like to add that the authors seem to use the term energy function instead of Lyapunov function. In my view an energy function (based on physical concepts) always exists for a system. However, it may not be a Lyapunov function. Incidentally, the concept behind equal area criterion is energy based and can be extended to multimachine systems with proper qualifications.

\section{References}

[A] K. R. Padiyar and H. S. Y. Sastry, "A Topological Energy Function Analysis of Stability of Power Systems," Int. J. Elec. Power and Energy Syst., vol. 9, no. 1, Jan. 1987, pp. 9-16.

[B] K. R. Padiyar and K. K. Ghosh, "Direct Stability Evaluation of Power Systems With Detailed Generator Models Using Structure-Preserving Energy Functions," Int. J. Elec. Power and Energy Syst., vol. 41, no. 1, Jan. 1989, pp. 47-55.

P. G. Murthy and M. Pavella (University of Liège, Liège, Belgium): This paper is an interesting outcome of the theoretical work carried out earlier by the authors (Refs. [14] and [31] of the paper) and we congratulate them.

The authors through their earlier investigations (Ref. [31] of the paper) set up a basis for the stability boundary containing the UEPs and further establish a relationship between the stability boundary of the second order (original) system and that of its associated gradient system (PEBS) cast in the reduced order space, the relative rotor angle subspace in this case (the fundamental basis for the BCU method). It may be recalled that the acronym PEBS was originally introduced and defined by Athay et al. [A]. Kakimoto et al. (Ref. [16] of the paper) earlier laid the necessary foundation for its characterization. PEBS refers collectively to a set of curves which are orthogonal to the equipotential curves (constant potential energy contours) and pass through the UEPs. It is a means of identifying possibly the controlling (or relevant or interesting) UEP for a given faulted system trajector to be used for subsequently computing the stability region (whatever the approach be of Kakimoto et al., Athay et al. or Tavora and Smith [B]).

In the procedure followed for the BCU method (version 1 or version 2) step 1 is used to detect the exit point of the faulted system trajectory on the stability boundary. If our understanding is right, this point is the same as the one proposed by Kakimoto et al., along with a procedure for its identification (Ref. [16] of the paper). Step 2 is aimed at the minimization of a scalar function (obtained by summing over all the $n$ generators in the system the squares of the power imbalance of each generator) on the stability boundary and towards a UEP. This is similar to 
the procedure given by Athay et al., (Ref. [17] of the paper). Step 3 (version 1) or step 4 (version 2) of the BCU method is equivalent to step 6 in the same reference of Athay et al.

The formulation of the gradient system and the related results of the BCU method were possible only when transfer conductances are neglected.

Our questions are the following.

(i) How well the BCU method may adapt itself to the practical case of power systems with transfer conductances, as in this case the $V$ function (eqn. 6 of the paper) is no more an energy function and does not satisfy the properties required for an energy function? Since the theoretical basis (Ref. [31] of the paper) cannot take into account transfer conductances, the concern is whether the point so reached in the process always turns out to be the actual UEP of interest.

(ii) As the proposed BCU method apparently is an off-shoot of that of Kakimoto it would be useful to compare their results with those provided by Kakimoto with respect to both efficiency and accuracy.

(iii) Two sets of integrations and one minimization have to be carried out apart from obtaining the solution of a set of power imbalance equations. We would like to know in this context how much computing effort is required by the BCU method to provide one critical clearing time.

Concerning the authors' reference to our previous work, we would like to observe the following.

(ii) The rare failures of the Kakimoto method reported in Ref. [26] of the paper (overoptimistic as well as overpessimistic diagnostics) were later on circumvented by the two "safeguards" proposed in Ref. [C]. We point it out again to be fair with the method.

(ii) The acceleration approach proposed earlier by us (Ref. [27] of the paper) cannot take into account transfer conductances. But when it comes to real life large power systems one has to consider transfer conductances, stringent operating conditions, and complex contingency scenarios. The various direct methods then cease to be reliable; besides, their computational burden increases substantially. An approach that properly tackles such stringent conditions is the extended equal area criterion (EEAC). This is a very simple method, but considers transfer conductances, complies with any stability scenarios, and proves to be extermely fast and accurate [D].

In conclusion we would like to mention that it had been the endeavor of several researchers in the past to obtain a realistic stability region that is both highly reliable (in terms of providing consistently a converging and relevant solution) and extremely effective (in terms of accuracy of results and speed). This paper is another attempt in this direction and it is hoped that the method stands the test of time.

\section{References}

[A] T. Athay, V. R. Sherkat, R. Podmore, S. Virmani, and C. Puech, "Transient energy stability analysis," Proc. Engineering Foundation Conference (conf-790904-P1) on Systems Engineering for Power: Emergency operating state control, Davos, Switzerland, Sept./Oct. 1979.

[B] C. J. Tavora and O. J. M. Smith, "Equilibrium analysis of power systems," as well as "Stability analysis of power systems," IEEE Trans. on PAS, Vol. PAS-91, May/June 1971, pp. 1131-1144.

[C] Th. Van Cutsem, B. Toumi, Y. Xue, M. Ribbens-Pavella, "Direct criteria for structure preserving models of electric power systems," IFAC Symposium on Power Systems and Power Plant Control, August 12-15, 1986, Beijing, China.

[D] Y. Xue, L. Wehenkel, R. Belhomme, P. Rousseaux, M. Pavella, E. Euxibie, B. Heilbronn, J. F. Lesigne, "Extended equal area criterion revisited," IEEE/PES 1991 Summer Meeting, Sand Diego, California, July 28-August 1.

B. Berggren and J. Bubenko (Royal Institute of Technology, Stockholm, Sweden): The authors are to be commended for their novel approach to the, in direct analysis of power system transient stability, central issue of finding the controlling u.e.p. for a particular disturbance. The authors comments on the following would be helpful:

In a stressed system, with 'large' transfer conductances, what is the relation between $\partial \mathrm{A}\left(\delta_{\mathrm{s}}\right)$ and the exit point $\delta^{*}$ (given that $\left.\delta^{*} \neq \delta_{\mathrm{co}}\right)$ ? There seems to be a risk of obtaining an unreliable starting point $\delta_{\mathrm{o}}^{*}$, for the subsequent search for $\delta_{\mathrm{co}}$, if $\delta^{*}$ is located too far from $\mathrm{W}^{\mathrm{s}}\left(\delta_{\mathrm{co}}\right)$.

V. VITTAL and A. A. FOUAD, Iowa State University, Ames, Iowa 50011. The authors are to be commended for an interesting paper on the development of an analytically based technique for the determination of the controlling UEP for direct transient stability assessment.

The discussors have used the proposed method with five modifications on several test systems. Most of the modification were needed to make the technique work for a relatively stressed power network. These modifications include:

i. Reformulation in the center of inertia frame of reference.

ii. Change of integration steps to build robustness and sensitivity into exit point determination.

iii. Use of a robust automatic step length selection ODE solver for the gradient system.

iv. Use of second derivative information for determination of the exact UEP.

v. Extension of the technique to include higher order machine models and effect of excitation.

Nearly sixty different cases were analyzed on four different systems ranging in size from 50-generators to 161-generators. Most of these systems are very stressed and demonstrate the inter-area mode of separation.

With respect to these cases the following observations are derived from our detailed numerical analysis. The gradient system equations in these cases are very stiff and illconditioned. A robust ODE solver with automatic step length selection is essential. The integration process detailed in Step 2 of the paper will not yield a first local minimum of $\sum_{i=1}^{n}\left\|f_{i}(\delta)\right\|$ unless a robust integration procedure is used. This has been experienced in all the systems analyzed.

In all cases of the inter-area mode situation, the initial guess for the controlling UEP, $\delta_{o}^{*}$, which is used as a starting point for the solution of the nonlinear algebraic equations to obtain the exact UEP appears to be quite far away from the controlling UEP in angle space. Furthermore, in order to converge to the controlling UEP a robust nonlinear equation solver which uses second derivative information is almost always necessary. To illustrate this aspect, the inter-area test 
case presented in the IEEE test system given in reference [A] is used. Table 1 shows the starting point $\delta_{o}^{*}$ for the controlling UEP using the BCU method obtained from step 2 and the exact UEP $\delta_{c o}^{*}$ obtained after solving the nonlinear algebraic equations for $\sum_{i=1}^{n}\left\|f_{i}(\delta)\right\|=0$ with $\delta_{0}^{*}$ as a starting point. The results in the table clearly show that in angle space the coordinates of the initial guess could be quite far away from the controlling UEP.

The theory developed in the paper indicates that $\delta_{o}^{*}$ obtained along the gradient system would be very close to the controlling UEP. Although, actual numerical results obtained by the discussors from several realistic systems show that $\delta_{c o}^{*}$ could be quite far away from the exact UEP $\delta_{c o}^{*}$, especially for the cases where the power network is stressed. Have the authors checked their proposed numerical technique on any power network exhibiting the interarea mode behavior, including the IEEE bench mark test system in reference [A]?

It is to be noted that $\delta_{o}^{*}$ in conjunction with a robust numerical technique provides a very good starting point for the controlling UEP. What analytical implications will such situations raise with regard to the statement in the paper that the reduced system trajectory will just pass by the controlling UEP? In such situations, how does one reconcile the theory with results obtained from simulation?

Table 1

COMPARISON BETWEEN $\delta_{0}^{*}$ and $\delta_{c o}^{*}$

\begin{tabular}{|c|c|c|}
\hline $\begin{array}{l}\text { Generator } \\
\text { No. }\end{array}$ & Initial Point for UEP Solution & $\begin{array}{c}\text { Exact UEP } \\
\delta_{c \ell}^{\circ} \\
\end{array}$ \\
\hline 1 & 34.32897 & 90.60786 \\
\hline 2 & 58.52708 & 116.92661 \\
\hline 3 & 58.41652 & 118.41063 \\
\hline 4 & 58.12256 & 118.52133 \\
\hline$s$ & 44.60900 & 112.35716 \\
\hline 6 & 87.12239 & 147.42142 \\
\hline 7 & 49.89610 & 111.23231 \\
\hline 8 & 54.92913 & 119.37025 \\
\hline 9 & 93.15012 & 154.89912 \\
\hline 10 & 43.10210 & 100.16379 \\
\hline 11 & 52.14607 & 94.95609 \\
\hline 12 & 66.15239 & 127.73028 \\
\hline 13 & 52.44674 & 122.59979 \\
\hline 14 & 94.83475 & 152.39862 \\
\hline 15 & 81.96613 & 149.61255 \\
\hline 16 & 75.91685 & 137.30670 \\
\hline 17 & 72.70155 & 130.89247 \\
\hline 18 & 35.98043 & 54.58508 \\
\hline 19 & 68.46889 & 133.63513 \\
\hline 20 & 153.37971 & 165.24542 \\
\hline 21 & 79.63909 & 138.64793 \\
\hline 22 & 78.63909 & 138.75079 \\
\hline 23 & 37.55813 & $\mathbf{9 8 . 7 6 7 1 1}$ \\
\hline 24 & 31.41294 & 99.95633 \\
\hline 25 & 94.95258 & 157.52249 \\
\hline 26 & 115.14426 & 151.26062 \\
\hline 27 & 71.87405 & 130.31325 \\
\hline 28 & -3.79213 & -3.17831 \\
\hline 29 & 0.11078 & 5.86947 \\
\hline 30 & 6.45139 & $\begin{array}{r}2.00941 \\
16.22913\end{array}$ \\
\hline 31 & 1.50834 & 9.14556 \\
\hline 32 & -43.12691 & -28.80369 \\
\hline 33 & 17.15852 & $\begin{array}{r}-21.80824 \\
71.80824\end{array}$ \\
\hline 34 & 36.76693 & 93.36178 \\
\hline 35 & 48.57814 & 105.14249 \\
\hline 36 & $\begin{array}{r}-20.83822 \\
\end{array}$ & 1.46311 \\
\hline 37 & -36.38322 & -25.64944 \\
\hline 38 & -12.17302 & -4.86038 \\
\hline $\begin{array}{l}30 \\
39\end{array}$ & 11.68494 & 27.25109 \\
\hline 40 & -5.67626 & -12.47140 \\
\hline 41 & 34.20311 & 28.14138 \\
\hline 42 & 9.46913 & $\begin{array}{r}2.14892 \\
2.1482\end{array}$ \\
\hline 43 & -72.11533 & -98.00967 \\
\hline 44 & -22.02707 & -39.82884 \\
\hline 45 & -1.49248 & -4.10896 \\
\hline 46 & 0.88079 & 3.50284 \\
\hline 47 & 4.65712 & 11.01902 \\
\hline 48 & 1.89332 & 5.04147 \\
\hline 49 & 15.36269 & 4.23330 \\
\hline 50 & -4.66088 & -19.82935 \\
\hline
\end{tabular}

[A] IEEE Committee Report, "Transient Stability Test Systems for Direct Stability Methods." Paper no. 91 WM 224-6-PWRS (Feb. 1991).

Manuscript received August 23, 1991.
Hsiao-Dong Chiang (Cornell University), Felix F. Wu and Pravin P. Varaiya (University of California, Berkeley).

We wish to thank the discussors for their interest in and thoughtful comments on this paper. We will respond to each discussor separately.

Given a power system stability model (which admits an energy function), the $\mathrm{BCU}$ method first explores the special structure of the underlying model with the aim to define an artificial, dimension-reduced system such that the following conditions are met:

\section{Static properties :}

- the locations of equilibrium points of the dimensionreduced system correspond to the locations of equilibrium points of the original system. For example, $\hat{x}$ is an e.p. of the dimension-reduced system if and only if $(\hat{x}, 0)$ is an e.p. of the original system, where $0 \in R^{m}, \mathrm{~m}$ is an appropriate positive integer.

- the types of equilibrium points of the dimensionreduced system are the same as that of the original system. For example, $x_{s}$ is a stable equilibrium point of the dimension-reduced system if and only if $\left(x_{s}, 0\right)$ is a stable equilibrium point of the original system.

Dynamical properties :

- there exists an energy function for the artificial, dimension-reduced system.

- an equilibrium point, say $x_{i}$, is on the stability boundary $\partial A\left(x_{s}\right)$ of the dimension-reduced system if and only if the equilibrium point $\left(x_{i}, 0\right)$ is on the stability boundary $\partial A\left(x_{s}, 0\right)$ of the original system.

- it is much easier to identify the stability boundary $\partial A\left(x_{s}\right)$ of the dimension-reduced system than to identify the stability boundary $\partial A\left(x_{s}, 0\right)$ of the original system.

Second, it finds the controlling u.e.p. of the dimensionreduced system by exploring the special structure of the stability boundary and the energy function of the dimension-reduced system. Third, it relates the controlling u.e.p. of the artificial system to the controlling u.e.p. of the original system. In summary, given a power system stability model, the fundamental ideas behind the BCU method can be roughly described as: (i) it explores the special structure of the underlying model so as to define an artificial, dimension-reduced system which captures all the equilibrium points on the stability boundary of the original system, and (ii) it finds the controlling u.e.p. of the original system via the controlling u.e.p. of the dimension-reduced system which is much easier to find than that of the origianl system. 
Therefore, given a power system stability model with certain properties, there exists a corresponding version of the $\mathrm{BCU}$ method. This gives a mathematical explanation of the artificial dimension-reduced system raised by Prof. Pai.

Dr. Berggren and Dr. Bubenko are correctly to point out the importance of finding the controlling u.e.p. for a given disturbance. The exit point $\delta^{*}$ is on the stability boundary $\partial A\left(\delta_{s}\right)$ of the artificial dimension-reduced system. This is the relation between $\delta^{*}$ and $\partial A\left(\delta_{s}\right)$. An unreliable starting point $\delta_{o}^{*}$ may occur if the exit point $\delta^{*}$ is located to far from $\partial A\left(\delta_{s}\right)$. The Step 1 of the BCU method is to detect the exit point $\delta^{*}$ at which the projected trajectory $\delta(t)$ exits the stability boundary of the dimension-reduction system. It was suggested in this paper that an effective computation scheme to implement Step 1 is : From the fault-on trajectory $(\delta(t), \omega(t))$, detect the exit point $\delta^{*}$ at which the projected trajectory $\delta(t)$ reaches the first local maximum of $V_{p}(\cdot)$.

The key role of direct methods for stability analysis can be stated as the following: given a (post-fault) nonlinear system described by a set of nonlinear equations with an initial condition (i.e. the state when the fault is cleared), determine based on energy function whether or not the ensuing trajectories will settle down to a desired steady-state without resort to explicit numerical integrations of the underlying nonlinear equations. Apparently, not every nonlinear system can be analyzed by using direct method; especially the class of nonlinear systems having limit cycles, or quasi-periodic solution, or chaotic attractors on their stability boundaries. We stress that direct methods in general, the controlling u.e.p. method in particular can only be applied to the class of nonlinear systems (nonlinear power system models) having an energy function. We say a function $V$ : $R^{n} \mapsto R$ is an energy function for a nonlinear system if the following three conditions are satisfied $[14, \mathrm{~A} 1]$ :

(i) the derivative of the energy function $V(x)$ along any system trajectory $\mathrm{x}(\mathrm{t})$ is non-positive, i.e.

$$
\dot{V}(x(t)) \leq 0
$$

(ii) If $\mathrm{x}(\mathrm{t})$ is a non-trivial trajectory (i.e. $\mathrm{x}(\mathrm{t})$ is not an equilibrium point (e.p.)), then there does not exist a time interval, say $\left[t_{1}, t_{2}\right], t_{2}>t_{1}$, such that $\dot{V}(x(t))=0$ for $t \in\left[t_{1}, t_{2}\right]$. Mathematically, this can be expressed as follows: along any non-trivial trajectory $\mathrm{x}(\mathrm{t})$ the set

$$
\{t \in R: \dot{V}(x(t))=0\}
$$

has measure zero in $\mathrm{R}$

(iii) If a trajectory $\mathrm{x}(\mathrm{t})$ has a bounded value of $\mathrm{V}(\mathrm{x}(\mathrm{t}))$ for $t \in R^{+}$, then this trajectory $\mathrm{x}(\mathrm{t})$ is also bounded. Stating this in brief : if $\mathrm{V}(\mathrm{x}(\mathrm{t}))$ is bounded, then $\mathrm{x}(\mathrm{t})$ is also bounded.

Property (i) indicates that the energy is non-increasing along its trajectory, but does not imply that the energy is strictly decreasing along its trajectory. There may exist a time interval $\left[t_{1}, t_{2}\right]$ such that $\dot{V}(x(t))=0$ for $t \in\left[t_{1}, t_{2}\right]$. Properties (i) and (ii) imply that the energy is strictly decreasing along any system trajectory. Property (iii) states that, along any system trajectory, the energy function is a proper map but its energy need not be a proper map for the entire state space. Obviously, an energy function is not a Lyapunov function. In this regard, we disagree with Dr. Padiyar that an energy function always exists for a system. We point out that an energy function always exists for a system not admitting any limit cycle, or quasi-periodic solution, or chaotic attractor in the system. A development of energy function theory can be found in [A1].

This paper does not assume any particular value for damping. Dr. Pai's question about the effects of using the COA formulation on the theoretical framework (of enrgy function, we pre-ssume). We offer the following. The equilibrium points of the system equation using the COA framework are non-hyperbolic (at least one of the eigenvalues is zero). A non-hyperbolic equilibrium point has an associated center manifold, in addition to its stable and unstable manifolds. In such a situation, the stability boundary of an asymptotically stable equilibrium point is contained in the union of the stable manifold as well as (part) of the center manifold of every equilibrium points on the stability boundary. The controlling u.e.p. method however is still applicable to direct analysis of transient stability using the COA formulation.

Traditionally, a majority of direct methods have been based on the network-reduction model where all the load representations are expressed in constant impedance and the network is reduced to the generator internal buses. As a result, the network-reduction model has the following critical shortcoming for the analysis of modern power systems: (i) in the context of system modeling, It precludes consideration of dynamic load behaviors (i.e. voltage and frequency variations) at load buses. (ii) in the context of physical explanation of results, reduction of the transmission network leads to loss of network topology and hence precludes study of transient energy shifts among different components of the entire power network. Network-preserving models (structure-preserving models) have been proposed in the last decade with an aim to overcome some of the shortcomings of the classical model and to improve the modeling of generators, exciters, automatic voltage regulators, and load representations. The first network-preserving model was developed by Bergen and Hill [A2], who assumed frequency dependent real power demands and constant reactive power demands. Narasimhamurthy 
and Musavi [A3] moved a step further by considering constant real power and voltage dependent reactive power loads. Padiyar and Sastry [A4] have included nonlinear voltage dependent loads for both real and reactive powers. Tsolas, Araposthasis and Varaiya [A5] developed a network-preserving model with the consideration of flux decay and constant real and reactive power loads. An energy function for a network-preserving model taking into account of static var compensators and their operating limits was developed by Hiskens and Hill [A6]. Therefore, we agree with Dr. Pardiyar the need to develop direct methods for network-preserving models with adequate load representations as well as its underlying theoretical foundations .

To Dr. Murthy and Dr. Pavella: All the simulation results presented in this paper using the $\mathrm{BCU}$ method are for power systems with transfer conductances. The BCU method is different from the method proposed by Athay et al. in [17]. Step 2 of the BCU method integrates the post-fault reduced system to find the first local minimum of $\sum_{i=1}^{n}\left\|f_{i}(\delta)\right\|$, say at $\delta_{o}^{*}$. The task involved in this step is numerical integration of the dimension-reduction system. This step does not require any minimization procedure. The method given by Athay et al. needs to perform an one-dimensional minimization procedure alnog the direction $\mathrm{h}=\Theta^{s s}-\Theta^{S E P 2}$ (see Steps 4 and 5 of the method given in [17]). Both the step 3 of the BCU method and the step 6 in [17] intend to find the exact location of a u.e.p. by solving a set of nonlinear algebraic equations. These two steps are different in that they use different initial points. The theoretical basis of the BCU method applies to power system models with transfer conductances; the details can be found in [A7]. The key point is the non-existence of an (exact) energy function for power system models with transfer conductances. A brief description of the development is as follows. By using one machine as reference, the (network-reduction) power system model can be transformed:

$$
\begin{gathered}
\delta_{1, n}=\omega_{1}-\omega_{n} \\
\vdots \\
\dot{\delta}_{n-1, n}=\omega_{n-1}-\omega_{n} \\
m_{1} \dot{\omega}_{1}=-d_{1} \omega_{1}+P_{m_{1}}-P e_{1}\left(\delta_{1, n}, \cdots, \delta_{n-1, n}\right) \\
\vdots \\
m_{n} \dot{\omega}_{n}=-d_{n} \omega_{n}+P_{m_{n}}-P e_{n}\left(\delta_{1, n}, \ldots, \delta_{n-1, n}\right)
\end{gathered}
$$

The above set of equations can be written in the following compact vector form

$$
\dot{\delta}=\left[\begin{array}{ll}
I_{n-1} & -e_{n-1}
\end{array}\right] \omega
$$

$$
M \dot{\omega}=-D \omega+\left[\begin{array}{c}
I_{n-1} \\
-e_{n-1}^{T}
\end{array}\right]\left[\begin{array}{c}
P_{m_{1}}-P_{e_{1}} \\
\vdots \\
\vdots \\
P_{m_{n-1}}-P_{e_{n-1}}
\end{array}\right]
$$

The BCU method explores the special structure of the underlying model so as to define an artificial, dimensionreduced system which aims to capture all the equilibrium points on the stability boundary of the underlying system and it finds the controlling u.e.p. of the original system via the controlling u.e.p. of the dimensionreduced system which is much easier to find than that of the origianl system. As to the network-reduction model, the following is a dimension-reduction system associate with the original system (1).

$$
\dot{\delta}=\left[\begin{array}{cl}
P_{m_{1}} & -P_{e_{1}} \\
& \vdots \\
& : \\
P_{m_{n-1}}- & P_{e_{n-1}}
\end{array}\right]
$$

To show the relationship between the artificial dimension-reduction system (2) and the original system (2), we proceed the following steps :

Step 1: Determine the static as well as dynamic relationship between the dimension-reduction system (2) and the following system

$$
\dot{\delta}=-\frac{\partial V_{p}(\delta)}{\partial \delta}
$$

$$
\begin{aligned}
& \text { where } \frac{\partial V_{p}(\delta)}{\partial \delta_{i}}= \\
& \sum_{i=1}^{n-1} \sum_{j=i+1}^{n} E_{i} E_{j} B_{i j} \sin \left(\delta_{i}-\delta_{j}\right)
\end{aligned}
$$

Step 2: Determine the static as well as dynamic relationship between the following systems

$$
\dot{\delta}=-\frac{\partial V_{p}(\delta)}{\partial \delta}
$$

and

$$
\begin{aligned}
\dot{\delta} & =-\frac{\partial V_{p}(\delta)}{\partial \delta} \\
M \dot{\omega} & =-D \omega
\end{aligned}
$$

Step 3: Determine the static as well as dynamic relationship between the following systems

$$
\begin{aligned}
\dot{\delta} & =-\frac{\partial V_{p}(\delta)}{\partial \delta} \\
M \dot{\omega} & =-D \omega
\end{aligned}
$$

and

$$
\begin{aligned}
\dot{\delta} & =\left[\begin{array}{ll}
I_{n-1} & -e_{n-1}
\end{array}\right] \omega \\
M \dot{\omega} & =-D \omega+\left[\begin{array}{c}
I_{n-1} \\
-e_{n-1}^{T}
\end{array}\right] \frac{\partial V_{p}(\delta)}{\partial \delta}
\end{aligned}
$$


Step 4: Determine the static as well as dynamic relationship between the following systems

$$
\begin{aligned}
\dot{\delta} & =\left[\begin{array}{ll}
I_{n-1} & -e_{n-1}
\end{array}\right] \omega \\
M \dot{\omega} & =-D \omega+\left[\begin{array}{c}
I_{n-1} \\
-e_{n-1}^{T}
\end{array}\right] \frac{\partial V_{p}(\delta)}{\partial \delta}
\end{aligned}
$$

and the original system which is

$$
\begin{aligned}
\dot{\delta} & =\left[\begin{array}{ll}
I_{n-1} & -e_{n-1}
\end{array}\right] \omega \\
M \dot{\omega} & =-D \omega+\left[\begin{array}{c}
I_{n-1} \\
-e_{n-1}^{T}
\end{array}\right]\left[\begin{array}{c}
P_{m_{1}}-P_{e_{1}} \\
\vdots \\
\vdots \\
P_{m_{n-1}}-P_{e_{n-1}}
\end{array}\right]
\end{aligned}
$$

In Step 1 and Step 4, we can show that, if the transfer conductance $G$ is sufficiently small, then

(Static relationship) $\left(\hat{\delta}_{x}\right)$ is a type-k equilibrium point of the dimension-reduction system (2) if and only if $\left(\hat{\delta}_{x}\right)$ is a type-k equilibrium point of the system $(3)$. $\left(\hat{\delta}_{x}, 0\right)$ is a type-k equilibrium point of the system (6) if and only if $\left(\hat{\delta}_{x}, 0\right)$ is a type-k equilibrium point of the original system (7).

(Dynamic relationship) $(\bar{\delta})$ is on the stability boundary $\partial A\left(\delta_{s}\right)$ of the dimension-reduction system (2) if and only $(\bar{\delta})$ is on the stability boundary $\partial A\left(\delta_{s}\right)$ of the system $(3) .(\bar{\delta}, 0)$ is on the stability boundary $\partial A\left(\delta_{s}, 0\right)$ of the system $(6)$ if and only $(\bar{\delta}$, $0)$ is on the stability boundary $\partial A\left(\delta_{s}, 0\right)$ of the original system ( 7 ).

In Step 2, it is easy to see that the following relationship also hold because the variables $\delta$ and $\omega$ of system (5) are decouped and the vector field of $\delta$ in system (5) is exactly the same as the vector field in system (3). Thus, we have the following results

(Static relationship) $\left(\hat{\delta}_{x}\right)$ is a type-k equilibrium point of the system (3) if and only if $\left(\hat{\delta}_{x}, 0\right)$ is a type-k equilibrium point of the system (5).

(Dynamic relationship) $(\bar{\delta})$ is on the stability boundary $\partial A\left(\delta_{s}\right)$ of the system $(3)$ if and only $(\bar{\delta}$, $0)$ is on the stability boundary $\partial A\left(\delta_{s}, 0\right)$ of the system (5).

In Step 3, we can show that $\left(\hat{\delta}_{x}, 0\right)$ is a type-k equilibrium point of the system (5) if and only if $\left(\hat{\delta}_{x}, 0\right)$ is a type-k equilibrium point of the system (6). Moreover, we can show that if an one-parameter transversality condition is satisfied, then

(Dynamic relationship) $(\bar{\delta}, 0)$ is on the stability boundary $\partial A\left(\delta_{s}, 0\right)$ of the system (5) if and only $(\bar{\delta}, 0)$ is on the stability boundary $\partial A\left(\delta_{s}, 0\right)$ of the system (6).
Thus, the static as well as dynamic relationship between the original system and the dimension-reduction system can be built up via step 1 through step 4 by connecting the relationship between the dimension-reduction system (2) and system (3) (Step 1), system (3) and system (5) (Step 2), system (5) and system (6) (Step 3), system (6) and system (7) (Step 4) (See Fig. 1). It can be shown [A7] that if zero is a regular value of $\frac{\partial V_{p}(\delta)}{\partial \delta_{i}}$, then there exists a positive number $\epsilon>0$ such that if the transfer conductance of system (1) satisfies $G_{i j}<\epsilon$, we have

(R1) $(\delta)$ is a type-k equilibrium point of the dimensionreduction system (2) if and only if $(\delta, 0)$ is a type$k$ equilibrium point of the original system (1). In particular, $\left(\delta_{s}\right)$ is a stable equilibrium point of the dimension-reduction system (2) if and only if $\left(\delta_{s}, 0\right)$ is a stable equilibrium point of the original system (1).

Moreover, if the intersections of the stable and unstable manifolds of the equilibrium points on the stability boundary $\partial A\left(\delta_{s}, 0\right)$ of the parameterized system $\mathrm{d}(\lambda)$ satisfy the transversality condition for $\lambda \in[0,1]$, then

[R2] the equilibrium point $\left(\delta_{i}, 0\right)$ is on the stability boundary $\partial A\left(\delta_{s}, 0\right)$ of system (1) if and only if the equilibrium point $\left(\delta_{i}\right)$ is on the stability boundary $\partial A\left(\delta_{s}\right)$ of system (2).

[R3]

$$
\begin{aligned}
\partial A\left(\delta_{s}, 0\right) & =\cup W^{s}\left(\delta_{i}, 0\right) \\
\partial A\left(\delta_{s}\right) & =\cup W^{s}\left(\delta_{i}\right)
\end{aligned}
$$

Result (R1) jusitifies the efforts of establishing the relationship between the stability region $A\left(\delta_{s}\right)$ of the dimension-reduction system (2) and the stability region $A\left(\delta_{s}, 0\right)$ of the original system (1). Results (R2) and (R3) establish a dynamic relationship between the stability boundary $\partial A\left(\delta_{s}\right)$ and the stability boundary $\partial A\left(\delta_{s}, 0\right)$ and suggests the plausibility of finding the controlling u.e.p. of the original system (1) via finding the controlling u.e.p. of the dimension-reduction system

As to the computational aspects of the BCU method, we offer the following. First, it should be pointed out that the $\mathrm{BCU}$ method does not require minimization procedures. Second, there are basically three major computational tasks in the BCU method: (i) compute the point with the first local maximum of potential energy along the projected fault-on trajectory, (ii) compute the trajectory of the post-fault dimensionreduction system, and (iii) compute the controlling u.e.p. via solving the nonlinear algebraic equations related to the dimension-reduction system. Task (i) is basically the PEBS precodure. 
The PEBS method (Kakimoto's method) is fast but is inconsistent in accuracy; it gives both over-estimate and under-estimate results (see the Table in the paper). We have similar experience with the PEBS method when applied to the test system with the network-preserving model. For example, for the case of a fault occurring at bus 6 with line 6-1 tripped, the PEBS method gives CCT of 0.13 second while the exact CCT, given by the time-domain method, is 0.08 second; an over-estimation result. for the case of a fault occurring at bus 6 with line 6-1 tripped, the PEBS method gives CCT of 0.13 second while the exact CCT, given by the time-domain method, is 0.08 second; an $(60 \%)$ over-estimate result. For the case of a fault occurring at bus 12 with line 12 14 tripped, the PEBS method gives CCT of 0.16 second while the exact CCT is 0.085 second; an $(90 \%)$ overestimation result.

The Step 2 of the BCU method presented in this paper reads 'Use the point $\delta^{*}$ as initial condition and integrate the post-fault reduced system to find the first local minimum of $\sum_{i=1}^{n}\left\|f_{i}(\delta)\right\|$, say at $\delta_{o}^{*}$. The comment by Dr. Vittal and Dr. Fouad 'The integration process detailed in Step 2 of the paper will not yield a first local minimum of $\sum_{i=1}^{n}\left\|f_{i}(\delta)\right\|$ unless a robust integration procedure is used' is unfounded. We remark in this paper that 'The reduced system can be stiff. In such a case, a stiff differential equation solver is recommended to implement Step 2.'. As to the Step 3 of the BCU method, we have been using Newton method to implement this step and our experience with Newton method is quite positive. The test system used in this paper is the IEEE bench mark test system [A8]. We have applied the BCU method with the proposed numerical technique to several faults on the IEEE bench mark system resulting interarea mode behaviors. We remark that the theory developed in this paper does not indicate that $\delta_{0}^{*}$ obtained along the gradient system would be very close to the controlling UEP. Note that the exit point $\delta^{*}$ must lie on the stable manifold of the controlling u.e.p. Step 2 of the BCU method gives a trajectory which would converge to the controlling u.e.p. as time increases. However, in practical computation, the point calculated in Step 1 can not be exactly the exit point but lies in a neighborhood of the exit point. Consequently, the resulting trajectory will just pass by the controlling u.e.p. according to the following general property of the trajectory regarding the dependence on initial conditions: consider the general (autonomous) nonlinear dynamical system described by equation $\dot{x}=f(x)$ which satisfies the existence and uniqueness condition and $\mathrm{f}(\mathrm{x})$ is $C^{r}$. Let the solution curve starting from $x_{0}$ at $\mathrm{t}=0$ be called a (system) trajectory $\Phi\left(t, x_{0}\right)$. Then $\Phi\left(t, x_{0}\right)$ is a $C^{r}$ function of $\mathrm{t}$ and $x_{0}$. One implication of this result is the comment by Dr. Vittal and Dr. Fouad, also indicated in the paper, that ' $\delta_{0}^{*}$ in conjunction with a robust numerical technique provides a very good starting point for the controlling UEP'.

The BCU method has been evaluated on a large-scale power system and compared favorably against other methods in terms of its reliability and computational efforts in $[A 9, A 10]$. The BCU method has been applied to the fast derivation of power transfer limit in [A11] and applied to real power rescheduling to increase dynamic security in [A12].

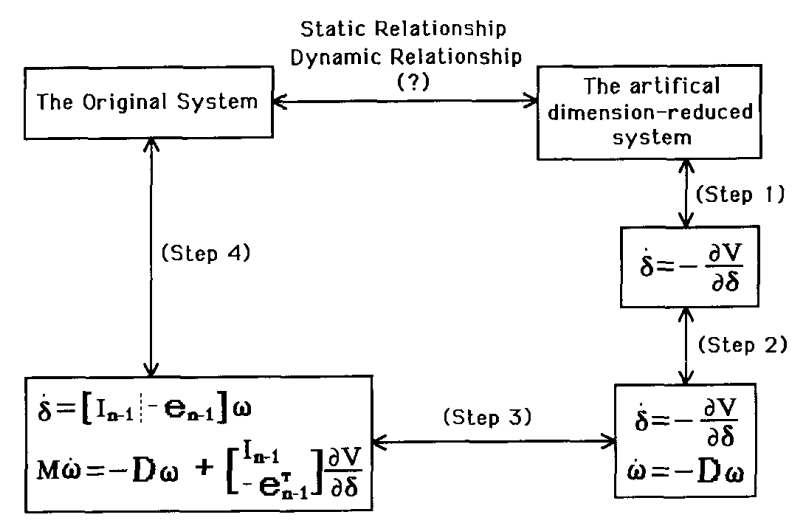

Figure I: Four steps to determine the static as well as dynamic relationships between the dimension-reduction systern and the original system

[A1] H.D. Chiang, "Analytical Results on Direct Methods for Power System Transient Stability Analysis", Advances in Control and Dynamic Systems, Volume XL, Theme: Advances in Electric Power and Energy Conversion Systems Dynamics and Control, Academic Press, Vol. 43, Part 3, pp. 275-334, 1991.

[A2] A. R. Bergen and D. J. Hill, ' A structure preserving model for power system stability analysis,' IEEE Trans on Power Apparatus and systems, Vol. PAS-100, January, 1981, pp.25-35.

[A3] N. Tsolas, A. Arapostathis and P.P. Varaiya, 'A structure preserving energy function for power system transient stability analysis,' IEEE Trans. on Circuits and Systems Vol. CAS-32, Oct. 1985, pp. 1041-1049.

[A4] N. Narasimhamurthi and M. R. Musavi, 'A general energy function for transient stability analysis of power systems,' IEEE Trans. on Circuits and Systems Vol. CAS-31 1984, pp.637-645.

[A5] K.R. Padiyar and K.K. Ghosh, 'Direct stability evaluation of power systems with detailed generator models using structure-preserving energy functions' International Journal of Electrical Power and Systems, Vol. 11, No. 1 , Jan. 1989, pp. 47-56.

[A6] I.A. Hiskens and D.J. Hill, 'Incorporation of SVCS into energy function methods', IEEE Trans. on Power Systems, Vol. 7, Feb. 1992, pp. 133-140. 
[A7] H.D. Chiang, "The BCU Method for Direct Stability Analysis of Electric Power Systems: Theory and Applications", pp. 1-50, to be published in 1994.

[A8] IEEE Committee Report, 'Transient Stability Test Systems for Direct Stability Methods', IEEE Trans. on Power Systems, Vol. 7, Feb. 1992, pp. 37-43.

[A9] F. A. Rahimi, 'Evaluation of transient energy function method software for dynamic security analysis', EPRI Project RP 4000-18, Final Report, Dec. 1990.
[A10] F. A. Rahimi, M.G. Lauby, J.N. Wrubel and K.L. Lee 'Evaluation of the transient energy function method for on-line dynamic security assessment', IEEE Transaction on Power Systems, Vol. 8, No. 2, May 1993, pp.497-507.

[A11] J. Tong, H.-D. Chiang and T.P. Conneen, 'A sensitivity-based BCU method for fast derivation of stability limits in electric power systems', to appear in IEEE Transaction on Power Systems.

[A12] D.H. Kuo and A. Bose, 'A generation rescheduling method to increase the dynamic security of power systems', to appear in IEEE Transactions on Power Systems. 